\title{
DISCRETE MULTIPLE HILBERT TYPE INEQUALITY WITH NON-HOMOGENEOUS KERNEL
}

\author{
Biserka Draščić Ban, Josip Pečarić, Ivan Perić, and Tibor Pogány
}

\begin{abstract}
Multiple discrete Hilbert type inequalities are established in the case of non-homogeneous kernel function by means of Laplace integral representation of associated Dirichlet series. Using newly derived integral expressions for the Mordell-Tornheim Zeta function a set of subsequent special cases, interesting by themselves, are obtained as corollaries of the main inequality.
\end{abstract}

\section{Introduction}

Let $\ell_{p}$ be the space of all complex sequences $\mathbf{x}=\left(x_{n}\right)_{n=1}^{\infty}$ with the finite norm $\|\mathbf{x}\|_{p}:=\left(\sum_{n=1}^{\infty}\left|x_{n}\right|^{p}\right)^{1 / p}$ endowed. Let $\mathbf{a}=\left(a_{n}\right)_{n=1}^{\infty} \in \ell_{p}, \mathbf{b}=\left(b_{n}\right)_{n=1}^{\infty} \in \ell_{q}$ be nonnegative sequences and $1 / p+1 / q=1, p>1$. Then

$$
\sum_{m, n \in \mathbb{N}} \frac{a_{m} b_{n}}{m+n}<\frac{\pi}{\sin (\pi / p)}\|\mathbf{a}\|_{p}\|\mathbf{b}\|_{q}
$$

where constant $\pi / \sin (\pi / p)$ is the best possible [3, p. 253].

This is the famous discrete Hilbert double series theorem or Hilbert inequality, a topic of interest of many mathematicians now-a-days too. The accustomary approach to deriving Hilbert's inequality is by applying the Hölder inequality to suitably transformed Hilbert type double sum expression, i.e., to the bilinear form

$$
\mathfrak{H}_{K}^{\mathbf{a}, \mathbf{b}}:=\sum_{m, n \in \mathbb{N}} K(m, n) a_{m} b_{n},
$$

where $\mathbf{a}, \mathbf{b}$ are nonnegative and $K(\cdot, \cdot)$ is the kernel function (of the double series (2)).

Received August 1, 2008.

2000 Mathematics Subject Classification. Primary 26D15; Secondary 40B05, 40 G99.

Key words and phrases. discrete Hilbert type inequality, discrete multiple Hilbert type inequality, Dirichlet-series, non-homogeneous kernel, homogeneous kernel, multiple Hölder inequality, Tornheim's double sum, Witten Zeta function, Mordell-Tornheim Zeta function.

The authors were supported in parts by the Ministry of Sciences, Education and Sports of Croatia under Research Project No. 112-2352818-2814 (Draščić Ban \& Pogány), 1171170889-0888 (Pečarić) and 058-1170889-1050 (Perić). 
For $p>1, p^{-1}+q^{-1}=1 ; r \in(3-\min \{p, q\}, 3], r^{-1}+s^{-1}=1$ and $\mathbf{n}^{(2-r) / p} \mathbf{a}^{r}:=\left(n^{(2-r) / p} a_{n}^{r}\right)_{n \geq 1} \in \ell_{p}, \mathbf{n}^{(2-r) / q} \mathbf{b}^{r} \in \ell_{q}$, Pogány [7] recently deduced

$$
\sum_{m, n \in \mathbb{N}} \frac{a_{m} b_{n}}{\left(\lambda_{m}+\rho_{n}\right)^{\mu}} \leq \mathbf{C}_{\boldsymbol{\lambda}, \boldsymbol{\rho}}\left\|\mathbf{n}^{(2-r) / p} \mathbf{a}^{r}\right\|_{p}^{1 / r}\left\|\mathbf{n}^{(2-r) / q} \mathbf{b}^{r}\right\|_{q}^{1 / r},
$$

where the constant

$$
\begin{aligned}
\mathrm{C}_{\boldsymbol{\lambda}, \boldsymbol{\rho}}= & \mathrm{C}_{\boldsymbol{\lambda}, \boldsymbol{\rho}}(p, q, r, s, \mu) \\
:= & \left(\begin{array}{c}
\mu s+1 \\
2
\end{array}\right)^{1 / s} \mathrm{~B}^{1 / r}(1+(r-3) / p, 1+(r-3) / q) \\
& \quad \times\left(\int_{\lambda_{1}}^{\infty} \int_{\rho_{1}}^{\infty} \frac{\left[\lambda^{-1}(x)\right]\left[\rho^{-1}(y)\right]\left(\left[\lambda^{-1}(x)\right]+\left[\rho^{-1}(y)\right]+2\right)}{(x+y)^{s \mu+2}} \mathrm{~d} x \mathrm{~d} y\right)^{1 / s}
\end{aligned}
$$

is the best possible. The conditions

$$
\int_{\lambda_{1}}^{\infty} \frac{\left(\lambda^{-1}(x)\right)^{2}}{x^{s \mu / 2+1}} \mathrm{~d} x<\infty, \quad \int_{\rho_{1}}^{\infty} \frac{\left(\rho^{-1}(x)\right)^{2}}{x^{s \mu / 2+1}} \mathrm{~d} x<\infty
$$

secure the finiteness of $\mathrm{C}_{\boldsymbol{\lambda}, \boldsymbol{\rho}}[7$, Theorem 1], $\mathrm{B}(\cdot, \cdot)$ denotes the Euler Betafunction and $\lambda, \rho: \mathbb{R}_{+} \mapsto \mathbb{R}_{+}$are monotone increasing positive functions such that

$$
\lim _{x \rightarrow \infty} \lambda(x)=\lim _{x \rightarrow \infty} \rho(x)=\infty,
$$

and their restrictions are $\left.\lambda\right|_{\mathbb{N}}=\boldsymbol{\lambda}=\left(\lambda_{n}\right)_{n=1}^{\infty},\left.\rho\right|_{\mathbb{N}}=\boldsymbol{\rho}=\left(\rho_{n}\right)_{n=1}^{\infty}$.

Let us define more general Hilbert type series. Let $m \in \mathbb{N}_{2}:=\{2,3, \ldots\}, \mathbf{a}_{j}$, $j=\overline{1, m}$ be nonnegative sequences and let $\lambda_{1}, \ldots, \lambda_{m}: \mathbb{R}_{+} \mapsto \mathbb{R}_{+}$be monotone increasing positive functions such that

$$
\lim _{x \rightarrow \infty} \lambda_{j}(x)=\infty \quad(j=\overline{1, m}),
$$

and their restrictions are $\left.\lambda_{j}\right|_{\mathbb{N}}=\boldsymbol{\lambda}_{j}=\left(\lambda_{j}\left(n_{j}\right)\right)_{n_{j}=1}^{\infty}$. Let us denote $\mathbf{n}:=$ $\left(n_{1}, \ldots, n_{m}\right)$ an $m$-dimensional positive integer index (running on $\left.\mathbb{N}^{m}\right)$. Then the multiple Hilbert-type series reads

$$
\mathfrak{H}_{m}(\rho, \mathbf{a}, \boldsymbol{\lambda}):=\sum_{\mathbf{n} \in \mathbb{N}^{m}} \frac{\prod_{j=1}^{m} a_{n_{j}}}{\left(\lambda_{1}\left(n_{1}\right)+\cdots+\lambda_{m}\left(n_{m}\right)+\rho\right)^{\mu}} \quad(\rho, \mu>0) .
$$

Our main goal is to derive a sharp upper bound to (7).

Here, and in what follows, $\mathcal{I}(x)=x$ denotes the identity function, $f^{-1}(x)$ stands for the inverse of some $f(x)$ and $[X],\{X\}$ are the integer part and the fractional part of some $X$. Furthermore, $\mathcal{D}_{\lambda}(x)$ will stand for the Laplace integral expression of the Dirichlet series $[8, \S 5]$

$$
\mathcal{D}_{\lambda}(x)=\sum_{n \in \mathbb{N}} a_{n} e^{-\lambda(n) x}=x \int_{0}^{\infty} e^{-x t}\left(\sum_{n=1}^{\left[\lambda^{-1}(t)\right]} a_{n}\right) \mathrm{d} t
$$


for positive monotone increasing $(\lambda(n))_{n=1}^{\infty}$ satisfying (6). The internal sum we find using the Euler-Maclaurin summation formula given in the form $[8, \S 6]$

$$
\sum_{j=k+1}^{\ell} a_{j}=\int_{k}^{\ell} \mathfrak{d}_{x} a(x) \mathrm{d} x \quad(k, \ell \in \mathbb{Z})
$$

where the differential operator $\mathfrak{d}_{x}:=1+\{x\} \frac{\partial}{\partial x}$.

\section{The main result}

Theorem 1. Assume $m \in \mathbb{N}_{2}, p_{1}^{-1}+\cdots+p_{m}^{-1}+q^{-1} \geq 1, \mu>0, \boldsymbol{\Phi}=$ $\left(\Phi_{1}, \ldots, \Phi_{m}\right), \Phi_{i}, j=\overline{1, m}$ positive monotone increasing functions and let $\mathbf{a}_{j}, j=\overline{1, m}$ be nonnegative sequences such that

$$
\left(\Phi_{j}^{1 / q}\left(n_{j}\right) a_{n_{j}}\right)_{n_{j}=1}^{\infty} \in \ell_{p_{j}} \quad(j=\overline{1, m})
$$

while $\boldsymbol{\lambda}=\left(\lambda_{1}, \ldots \lambda_{m}\right)$ and all $\lambda_{j}$ satisfies (5). Then we have

$$
\mathfrak{H}_{m}(\rho, \mathbf{a}, \boldsymbol{\lambda}) \leq \mathrm{C}_{\mu, q}^{\mathbf{\Phi}}(\mathbf{a}, \boldsymbol{\lambda})\left\|\mathbf{a}_{1} \boldsymbol{\Phi}_{1}^{1 / q}\right\|_{p_{1}} \cdots\left\|\mathbf{a}_{m} \boldsymbol{\Phi}_{m}^{1 / q}\right\|_{p_{m}},
$$

where the constant factor equals

$$
\mathbf{C}_{\mu, q}^{\mathbf{\Phi}}(\mathbf{a}, \boldsymbol{\lambda})=\left(\left(\begin{array}{c}
\mu q+1 \\
2
\end{array}\right) \int_{\boldsymbol{\lambda}(\mathbf{1})}^{\infty} \int_{0}^{\left[\boldsymbol{\lambda}^{-1}(\mathbf{t})\right]} \frac{\prod_{j=1}^{m} \mathfrak{d}_{u_{j}}\left(1 / \Phi_{j}\left(u_{j}\right)\right)}{\left(t_{1}+\cdots+t_{m}+\rho\right)^{\mu q+2}} \mathbf{d t d} \mathbf{d u}\right)^{1 / q}
$$

Here $\int_{\boldsymbol{\lambda}(\mathbf{1})}^{\infty}:=\int_{\lambda_{1}(1)}^{\infty} \cdots \int_{\lambda_{m}(1)}^{\infty} ; \int_{0}^{\left[\boldsymbol{\lambda}^{-1}(\mathbf{t})\right]}:=\int_{0}^{\left[\lambda_{1}^{-1}\left(t_{1}\right)\right]} \cdots \int_{0}^{\left[\lambda_{m}^{-1}\left(t_{m}\right)\right]}$ stand as the suitable abbreviations for $m$-tuple integrals, while $\mathbf{d x}:=\mathrm{d} x_{1} \cdots \mathrm{d} x_{m}$.

Proof. Assume $q>1$ and rewrite the $m$-tuple sum into the form:

$$
\mathfrak{H}_{m}(\rho, \mathbf{a}, \boldsymbol{\lambda})=\sum_{\mathbf{n} \in \mathbb{N}^{m}} \frac{\Phi_{1}^{1 / q}\left(n_{1}\right) \cdots \Phi_{m}^{1 / q}\left(n_{m}\right) a_{n_{1}} \cdots a_{n_{m}}}{\Phi_{1}^{1 / q}\left(n_{1}\right) \cdots \Phi_{m}^{1 / q}\left(n_{m}\right)\left(\lambda_{1}\left(n_{1}\right)+\cdots+\lambda_{m}\left(n_{m}\right)+\rho\right)^{\mu}} .
$$

Then, making use of the generalized Hölder inequality with $p_{1}^{-1}+\cdots+p_{m}^{-1}+$ $q^{-1} \geq 1([4],[6])$, we get

$$
\begin{aligned}
\mathfrak{H}_{m}(\rho, \mathbf{a}, \boldsymbol{\lambda}) \leq & \left(\sum_{n_{1} \in \mathbb{N}} a_{n_{1}}^{p_{1}} \Phi_{1}^{p_{1} / q}\left(n_{1}\right)\right)^{1 / p_{1}} \cdots\left(\sum_{n_{m} \in \mathbb{N}} a_{n_{r}} \Phi_{m}^{p_{m} / q}\left(n_{m}\right)\right)^{1 / p_{m}} \\
& \times\left(\sum_{\mathbf{n} \in \mathbb{N}^{m}} \frac{1}{\Phi_{1}\left(n_{1}\right) \cdots \Phi_{m}\left(n_{m}\right)\left(\lambda_{1}\left(n_{1}\right)+\cdots+\lambda_{m}\left(n_{m}\right)+\rho\right)^{\mu q}}\right)^{1 / q}:=R .
\end{aligned}
$$


Transforming the general term of the multiple series by the Gamma function, we conclude

$$
\begin{aligned}
R & =\prod_{j=1}^{m}\left\|\mathbf{a}_{j} \boldsymbol{\Phi}_{j}^{1 / q}\right\|_{p_{j}}\left(\sum_{\mathbf{n} \in \mathbb{N}^{m}} \frac{1}{\Phi_{1}\left(n_{1}\right) \cdots \Phi_{m}\left(n_{m}\right)\left(\rho+\sum_{j=1}^{m} \lambda_{j}\left(n_{j}\right)\right)^{\mu q}}\right)^{1 / q} \\
& =\frac{\prod_{j=1}^{m}\left\|\mathbf{a}_{j} \boldsymbol{\Phi}_{j}^{1 / q}\right\|_{p_{j}}}{\Gamma^{1 / q}(\mu q)}\left(\int_{0}^{\infty} x^{\mu q-1} e^{-\rho x} \prod_{j=1}^{m}\left(\sum_{n_{j} \in \mathbb{N}} \frac{e^{-\lambda_{j}\left(n_{j}\right) x}}{\Phi_{j}\left(n_{j}\right)}\right) \mathrm{d} x\right)^{1 / q}
\end{aligned}
$$

Now, we apply the Laplace integral result (8) to all Dirichlet series

$$
\mathcal{D}_{\lambda_{j}}(x)=\sum_{n_{j} \in \mathbb{N}} \frac{e^{-\lambda_{j}\left(n_{j}\right) x}}{\Phi_{j}\left(n_{j}\right)}=x \int_{\lambda_{j}(1)}^{\infty} e^{-x t_{j}}\left(\sum_{n_{j}=1}^{\left[\lambda_{j}^{-1}\left(t_{j}\right)\right]} \frac{1}{\Phi_{j}\left(n_{j}\right)}\right) \mathrm{d} t_{j},
$$

and calculate the inner-most counting sum by the Euler-Maclaurin summation formula (9). We conclude

$$
\begin{aligned}
\mathcal{D}_{\lambda_{j}}(x) & =x \int_{\lambda_{j}(1)}^{\infty} e^{-x t_{j}}\left(\int_{0}^{\left[\lambda_{j}^{-1}\left(t_{j}\right)\right]} \mathfrak{d}_{u_{j}}\left(1 / \Phi_{j}\left(u_{j}\right)\right) \mathrm{d} u_{j}\right) \mathrm{d} t_{j} \\
& =x \int_{\lambda_{j}(1)}^{\infty} \int_{0}^{\left[\lambda_{j}^{-1}\left(t_{j}\right)\right]} e^{-x t_{j}} \mathfrak{d}_{u_{j}}\left(1 / \Phi_{j}\left(u_{j}\right)\right) \mathrm{d} t_{j} \mathrm{~d} u_{j} .
\end{aligned}
$$

Therefore, we easily get

$$
\begin{aligned}
\mathfrak{H}_{m}(\rho, \mathbf{a}, \boldsymbol{\lambda}) \leq & \frac{\left\|\mathbf{a}_{1} \boldsymbol{\Phi}_{1}^{1 / q}\right\|_{p_{1}} \cdots\left\|\mathbf{a}_{m} \boldsymbol{\Phi}_{m}^{1 / q}\right\|_{p_{m}}}{\Gamma^{1 / q}(\mu q)} \\
& \times\left(\int_{\boldsymbol{\lambda}(\mathbf{1})}^{\infty} \int_{0}^{\left[\boldsymbol{\lambda}^{-1}(\mathbf{t})\right]} \mathfrak{d}_{u_{1}}\left(\frac{1}{\Phi_{1}\left(u_{1}\right)}\right) \cdots \mathfrak{d}_{u_{m}}\left(\frac{1}{\Phi_{m}\left(u_{m}\right)}\right)\right. \\
& \left.\times\left(\int_{0}^{\infty} e^{-\left(t_{1}+\cdots+t_{m}+\rho\right) x} x^{\mu q+1} \mathrm{~d} x\right) \mathrm{d} t_{1} \cdots \mathrm{d} t_{m} \mathrm{~d} u_{1} \cdots \mathrm{d} u_{m}\right)^{1 / q} .
\end{aligned}
$$

Now, the right hand bound becomes

$$
\begin{aligned}
R= & \frac{\Gamma^{1 / q}(\mu q+2)}{\Gamma^{1 / q}(\mu q)}\left\|\mathbf{a}_{1} \boldsymbol{\Phi}_{1}^{1 / q}\right\|_{p_{1}} \cdots\left\|\mathbf{a}_{m} \boldsymbol{\Phi}_{m}^{1 / q}\right\|_{p_{m}} \\
& \times\left(\int_{\boldsymbol{\lambda}(\mathbf{1})}^{\infty} \int_{0}^{\left[\boldsymbol{\lambda}^{-1}(\mathbf{t})\right]} \frac{\prod_{j=1}^{m} \mathfrak{d}_{u_{j}}\left(1 / \Phi_{j}\left(u_{j}\right)\right)}{\left(t_{1}+\cdots+t_{m}+\rho\right)^{\mu q+2}} \mathbf{d t} \mathbf{d u}\right)^{1 / q} .
\end{aligned}
$$

Since all above involved series are convergent, the associated integral expressions are convergent as well. So, all interchanges of integration order are enabled. Reducing the last expression, we finish the proof. 


\section{Integral representation of Mordell-Tornheim Zeta-function}

In 1950 Tornheim [10] introduced the double series

$$
T(p, q, r)=\sum_{\mathbf{n} \in \mathbb{N}^{2}} \frac{1}{n_{1}^{p} n_{2}^{q}\left(n_{1}+n_{2}\right)^{r}} \quad(p, q, r>0, r+\min \{p, q\}>1) .
$$

In honor to the author it is called Tornheim double series, also known in literature as Witten Zeta function or Mordell series. Later, a various continuations of the domain for the function $T(p, q, r)$ are given, but here we are interested in the case $p>1, q>1, r>0$, see, for example, [1], [2], [9], [11], [12].

Matsumoto [5] introduced the related, so-called Mordell-Tornheim m-tuple Zeta-function in the form

$$
\zeta_{M T, m}(\mathbf{r} ; s)=\sum_{\mathbf{n} \in \mathbb{N}^{m}} \frac{1}{n_{1}^{r_{1}} \cdots n_{m}^{r_{m}}\left(n_{1}+\cdots+n_{m}\right)^{s}},
$$

where $\mathbf{r}:=\left(r_{1}, \ldots, r_{m}\right) ; r_{j}, s \in \mathbb{C}$. The series (16) is absolutely convergent for $\Re\left\{r_{j}\right\}>1, j=\overline{1, m}$ and $\Re\{s\}>0$. It is obvious that taking $m=2$ and real parameters (16) one reduces to (15).

Our goal is here to obtain an integral representation for the real parameter Mordell-Tornheim Zeta $\zeta_{M T, m}$. We give two different kind integral expressions for this special function.

Theorem 2. Let $r_{1}>1, \ldots, r_{m}>1, s>0$. Then the series $\zeta_{M T, m}(\mathbf{r} ; s)$ has the following integral representation:

$$
\zeta_{M T, m}(\mathbf{r} ; s)=\frac{1}{2^{m} \Gamma(s) \prod_{j=1}^{m} \Gamma\left(r_{j}\right)} \int_{\mathbb{R}_{+}^{m+1}} \frac{x^{s-1}}{e^{\frac{m x+t_{1}+\cdots+t_{m}}{2}}} \prod_{j=1}^{m} \frac{t_{j}^{r_{j}-1}}{\sinh \frac{x+t_{j}}{2}} \mathrm{~d} x \mathbf{d t} .
$$

Proof. First, let us rewrite $\zeta_{M T, m}$ into

$$
\zeta_{M T, m}\left(r_{1}, \ldots, r_{m} ; s\right)=\frac{1}{\Gamma(s)} \int_{0}^{\infty} x^{s-1} \prod_{j=1}^{m}\left(\sum_{n_{j} \in \mathbb{N}} \frac{e^{-n_{j} x}}{n_{j}^{r_{j}}}\right) \mathrm{d} x .
$$

Then the inner sums become

$$
\begin{aligned}
\sum_{n_{j} \in \mathbb{N}} \frac{e^{-n_{j} x}}{n_{j}^{r_{j}}} & =\frac{1}{\Gamma\left(r_{j}\right)} \int_{0}^{\infty} t_{j}^{r_{j}-1} \sum_{n_{j} \in \mathbb{N}} e^{-\left(t_{j}+x\right) n_{j}} \mathrm{~d} t_{j} \\
& =\frac{1}{\Gamma\left(r_{j}\right)} \int_{0}^{\infty} \frac{t_{j}^{r_{j}-1}}{e^{x+t_{j}}-1} \mathrm{~d} t_{j} \\
& =\frac{1}{2 \Gamma\left(r_{j}\right)} \int_{0}^{\infty} \frac{t^{t_{j}-1} e^{-\frac{x+t_{j}}{2}}}{\sinh \frac{x+t_{j}}{2}} \mathrm{~d} t_{j}
\end{aligned}
$$

Since all above series are convergent, their integral expressions converge simultaneously. The integration order interchanges are legitimate. Thus, straightforward steps result in the desired relation (17). 
Now, taking $m=2$ we get the integral representation for the TornheimWitten Zeta function.

Corollary 1. Let $p>1, q>1, r>0$. Then the series $T(p, q, r)$ possesses the integral representation:

$$
T(p, q, r)=\frac{1}{4 \Gamma(p) \Gamma(q) \Gamma(r)} \int_{0}^{\infty} \int_{0}^{\infty} \int_{0}^{\infty} \frac{x^{r-1} t_{1}^{p-1} t_{2}^{q-1} e^{-x-\frac{t_{1}+t_{2}}{2}}}{\sinh \frac{x+t_{1}}{2} \sinh \frac{x+t_{2}}{2}} \mathrm{~d} x \mathrm{~d} t_{1} \mathrm{~d} t_{2} .
$$

Another kind integral expression can be given specifying $\Phi_{j}\left(n_{j}\right)=n_{j}^{r_{j}}$, $\lambda_{j}\left(n_{j}\right)=n_{j}, j=\overline{1, m} ; \mu q=s$ in (12). Then, by means of (13)-(14) we deduce the following result.

Theorem 3. Let the situation be the same as then in the previous theorem. Then we have

$$
\zeta_{M T, m}\left(r_{1}, \ldots, r_{m} ; s\right)=\frac{s(s+1)}{2} \int_{\mathbf{1}}^{\infty} \int_{0}^{[\mathbf{t}]} \frac{\prod_{j=1}^{m} \mathfrak{d}_{u_{j}}\left(u_{j}^{-r_{j}}\right)}{\left(t_{1}+\cdots+t_{m}\right)^{s+2}} \mathbf{d t d} \mathbf{u}
$$

where the notations coincide with the ones in (10).

If we recall the proof of Theorem 3 and specify $m=2$, we get a new integral representation of the Tornheim series $T(p, q, r)$.

Corollary 2. Let $p, q, r>0, r+\min \{p, q\}>1$. Then the series (15) possesses the following integral expression

$$
T(p, q, r)=\left(\begin{array}{c}
r+1 \\
2
\end{array}\right) \int_{1}^{\infty} \int_{1}^{\infty} \int_{0}^{\left[t_{1}\right]} \int_{0}^{\left[t_{2}\right]} \frac{\mathfrak{d}_{u_{1}}\left(u_{1}^{-p}\right) \mathfrak{d}_{u_{2}}\left(u_{2}^{-q}\right)}{\left(t_{1}+t_{2}\right)^{r+2}} \mathrm{~d} t_{1} \mathrm{~d} t_{2} \mathrm{~d} u_{1} \mathrm{~d} u_{2} .
$$

\section{Special cases}

In this chapter we return to our multiple Hilbert-type series in which specifying step-by-step the functions $\Phi_{j}, \lambda_{j}, j=\overline{1, m}$ we get various special cases for $\mathfrak{H}_{m}(\rho, \mathbf{a}, \boldsymbol{\lambda})$ and its upper bounds.

\section{1. $\Phi_{j}\left(n_{j}\right)=n_{j}^{r_{j}}, r_{j}>1$}

Following the proving procedure of Theorem 1, we conclude

$$
\begin{aligned}
& \mathfrak{H}_{m}(\rho, \mathbf{a}, \boldsymbol{\lambda}) \\
\leq & \left(\sum_{n_{1} \in \mathbb{N}} a_{n_{1}} n_{1}^{p_{1} r_{1} / q}\right)^{1 / p_{1}} \cdots\left(\sum_{n_{m} \in \mathbb{N}} a_{n_{m}} n_{m}^{p_{m} r_{m} / q}\right)^{1 / p_{m}} \\
& \times\left(\sum_{\mathbf{n} \in \mathbb{N}^{m}} \frac{1}{n_{1}^{r_{1}} \cdots n_{m}^{r_{m}}\left(\lambda_{1}\left(n_{1}\right)+\cdots+\lambda_{m}\left(n_{m}\right)+\rho\right)^{\mu q}}\right)^{1 / q}
\end{aligned}
$$




$$
\begin{aligned}
& =\prod_{j=1}^{m}\left\|\mathbf{a}_{j} \mathbf{n}_{j}^{r_{j} / q}\right\|_{p_{j}}\left(\sum_{\mathbf{n} \in \mathbb{N}^{m}} \frac{1}{n_{1}^{r_{1}} \cdots n_{m}^{r_{m}}\left(\lambda_{1}\left(n_{1}\right)+\cdots+\lambda_{m}\left(n_{m}\right)+\rho\right)^{\mu q}}\right)^{1 / q} \\
& =\frac{\prod_{j=1}^{m}\left\|\mathbf{a}_{j} \mathbf{n}_{j}^{r_{j} / q}\right\|_{p_{j}}}{\Gamma^{1 / q}(\mu q)}\left(\int_{0}^{\infty} x^{\mu q-1} e^{-\rho x}\left(\prod_{j=1}^{m} \sum_{n_{j} \in \mathbb{N}} \frac{e^{-\lambda_{j}\left(n_{j}\right) x}}{n_{j}^{r_{j}}}\right) \mathrm{d} x\right)^{1 / q} .
\end{aligned}
$$

Now, as

$$
\mathcal{D}_{\lambda_{j}}(x)=\sum_{n_{j}=1}^{\infty} \frac{e^{-\lambda_{j}\left(n_{j}\right) x}}{n_{j}^{r_{j}}}=x \int_{\lambda_{j}(1)}^{\infty} e^{-x t_{j}}\left(\sum_{n_{j}=1}^{\left[\lambda_{j}^{-1}\left(t_{j}\right)\right]} n_{j}^{-r_{j}}\right) \mathrm{d} t_{j},
$$

by (9) we have

$$
\mathcal{D}_{\lambda_{j}}(x)=x \int_{\lambda_{j}(1)}^{\infty} \int_{0}^{\left[\lambda_{j}^{-1}\left(t_{j}\right)\right]} e^{-x t_{j}} \mathfrak{d}_{u_{j}}\left(u_{j}^{-r_{j}}\right) \mathrm{d} t_{j} \mathrm{~d} u_{j}
$$

So, we easily deduce the subsequent result.

Corollary 3. Assume $m \in \mathbb{N}_{2}, q>1, p_{1}^{-1}+\cdots+p_{m}^{-1}+q^{-1} \geq 1, \mu>0$ and let $\mathbf{a}_{j}$ be nonnegative sequences such that

$$
\left(n_{j}^{r_{i} / q} a_{n_{j}}\right)_{n_{j} \in \mathbb{N}} \in \ell_{p_{j}} \quad(j=\overline{1, m})
$$

with a conveniently chosen $\mathbf{r}:=\left(r_{1}, \ldots, r_{m}\right)$. When $\lambda_{1}, \ldots, \lambda_{m}: \mathbb{R}_{+} \mapsto \mathbb{R}_{+}$are positive monotone increasing functions such that satisfy (5), we have

$$
\sum_{\mathbf{n} \in \mathbb{N}^{m}} \frac{a_{n_{1}} \cdots a_{n_{m}}}{\left(\rho+\sum_{j=1}^{m} \lambda_{j}\left(n_{j}\right)\right)^{\mu}} \leq \mathrm{C}_{\mu, q}^{\mathbb{N}^{m}}(\mathbf{a}, \boldsymbol{\lambda})\left\|\mathbf{a}_{1} \mathbf{n}_{1}^{r_{1} / q}\right\|_{p_{1}} \cdots\left\|\mathbf{a}_{m} \mathbf{n}_{m}^{r_{m} / q}\right\|_{p_{m}},
$$

where the constant factor equals

(22) $\mathrm{C}_{\mu, q}^{\mathbb{N}^{\mathrm{m}}}(\mathbf{a}, \boldsymbol{\lambda})=\left(\left(\begin{array}{c}\mu q+1 \\ 2\end{array}\right) \int_{\boldsymbol{\lambda}(\mathbf{1})}^{\infty} \int_{0}^{\left[\boldsymbol{\lambda}^{-1}(\mathbf{t})\right]} \frac{\prod_{j=1}^{m} \mathfrak{d}_{u_{j}}\left(u_{j}^{-p_{j}}\right)}{\left(\rho+\sum_{j=1}^{m} t_{j}\right)^{\mu q+2}} \mathbf{d t d} \mathbf{u}\right)^{1 / q}$,

making use of abbreviations from Theorem 1.

4.2. $\Phi_{j}\left(n_{j}\right)=n_{j}^{r_{j}}, r_{j}>0, \lambda_{j}(x)=\mathcal{I}(x)=x, \rho=0$

In this case we get

$$
\begin{aligned}
\mathfrak{H}_{m}(0, \mathbf{a}, \mathcal{I}) & \leq\left(\sum_{\mathbf{n} \in \mathbb{N}_{m}} \frac{1}{n_{1}^{r_{1}} \cdots n_{m}^{r_{m}}\left(n_{1}+\cdots+n_{m}\right)^{\mu q}}\right)^{1 / q} \prod_{j=1}^{m}\left\|\mathbf{a}_{j} \mathbf{n}_{j}^{r_{j} / q}\right\|_{p_{j}} \\
& =\left\|\mathbf{a}_{1} \mathbf{n}_{1}^{r_{1} / q}\right\|_{p_{1}} \cdots\left\|\mathbf{a}_{m} \mathbf{n}_{m}^{r_{m} / q}\right\|_{p_{m}}\left(\zeta_{M T, r}\left(r_{1}, \ldots, r_{m} ; \mu q\right)\right)^{1 / q} .
\end{aligned}
$$

Now, there are two possible approaches to estimate the series $\mathfrak{H}_{m}(0, \mathbf{a}, \mathcal{I})$. First, we apply the integral expression (17) for the Mordell-Tornheim Zeta function $\zeta_{M T, r}$ achieved by Theorem 2 . 
544 BISERKA DRAŠČIĆ BAN, JOSIP PEČARIĆ, IVAN PERIĆ, AND TIBOR POGÁNY

Corollary 4. Let $m \in \mathbb{N}_{2}, q>1, p_{1}^{-1}+\cdots+p_{m}^{-1}+q^{-1} \geq 1, \mu>0, \mathbf{a}_{j}$ be nonnegative sequences such that $\left(n_{j}^{r_{j} / q} a_{n_{j}}\right)_{n_{j} \in \mathbb{N}} \in \ell_{p_{j}}, j=\overline{1, m}$ where $\mu q+$ $\min _{1 \leq j \leq m}\left\{r_{j}\right\}>1$. Then we have

$$
\mathfrak{H}_{m}(0, \mathbf{a}, \mathcal{I})=\sum_{\mathbf{n} \in \mathbb{N}^{m}}^{\infty} \frac{a_{n_{1}} \cdots a_{n_{m}}}{\left(n_{1}+\cdots+n_{m}\right)^{\mu}} \leq C_{\mu, q}^{\mathbb{N}^{\mathbf{m}}}(\mathbf{a}, \mathcal{I}) \prod_{j=1}^{m}\left\|\mathbf{a}_{j} \mathbf{n}_{j}^{r_{j} / q}\right\|_{p_{j}},
$$

where

$$
\mathrm{C}_{\mu, q}^{\mathbb{N}^{\mathbf{m}}}(\mathbf{a}, \mathcal{I})=\left(\frac{2^{-m}}{\Gamma(\mu q) \prod_{j=1}^{m} \Gamma\left(r_{j}\right)} \int_{\mathbb{R}_{+}^{m+1}} \frac{x^{\mu q-1}}{e^{\frac{m x+t_{1}+\cdots+t_{m}}{2}}} \prod_{i=1}^{m} \frac{t_{i}^{p_{i}-1}}{\sinh \frac{x+t_{i}}{2}} \mathrm{~d} x \mathbf{d t}\right)^{1 / q}
$$

with the use of notations from Theorem 1.

Second, we can apply the calculated integral representation (21) for $\zeta_{M T, m}$ exposed in Theorem 3.

Corollary 5. Suppose $m \in \mathbb{N}_{2}, q>1, p_{1}^{-1}+\cdots+p_{m}^{-1}+q^{-1} \geq 1, \mu>0, \mathbf{a}_{j}$ are nonnegative sequences such that $\left\|\mathbf{a}_{j} \mathbf{n}_{j}^{r_{j} / q}\right\|_{p_{j}} \in \ell_{p_{j}}, j=\overline{1, m}$. Then we have

$$
\mathfrak{H}_{m}(0, \mathbf{a}, \mathcal{I})=\sum_{\mathbf{n} \in \mathbb{N}^{m}}^{\infty} \frac{a_{n_{1}} \cdots a_{n_{m}}}{\left(n_{1}+\cdots+n_{m}\right)^{\mu}} \leq \mathrm{K}_{\mu, q}^{\mathbb{N}^{m}}(\mathbf{a}, \mathcal{I}) \prod_{j=1}^{m}\left\|\mathbf{a}_{j} \mathbf{n}_{j}^{r_{j} / q}\right\|_{p_{j}},
$$

where the constant factor equals

$$
\mathrm{K}_{\mu, q}^{\mathbb{N}^{\mathbf{m}}}(\mathbf{a}, \mathcal{I})=\left(\left(\begin{array}{c}
\mu q+1 \\
2
\end{array}\right) \int_{\mathbf{1}}^{\infty} \int_{0}^{[\mathbf{t}]} \frac{\prod_{j=1}^{m} \mathfrak{d}_{u_{j}}\left(u_{j}^{-p_{j}}\right)}{\left(t_{1}+\cdots+t_{m}\right)^{\mu q+2}} \mathbf{d t d} \mathbf{u}\right)^{1 / q}
$$

with the use of abbreviations analogous to ones in Theorem 1.

Proof. Consider the starting inequality (23). Now, we have

$$
\begin{aligned}
\mathfrak{H}_{m}(0, \mathbf{a}, \boldsymbol{I}) \leq & \frac{\left\|\mathbf{a}_{1} \mathbf{n}_{1}^{r_{1} / q}\right\|_{p_{1}} \cdots\left\|\mathbf{a}_{m} \mathbf{n}_{r}^{r_{m} / q}\right\|_{p_{m}}}{\Gamma^{1 / q}(\mu q)} \\
& \times\left(\int_{0}^{\infty} x^{\mu q-1}\left(\sum_{n_{1} \in \mathbb{N}} \frac{e^{-n_{1} x}}{n_{1}^{r_{1}}}\right) \cdots\left(\sum_{n_{m} \in \mathbb{N}} \frac{e^{-n_{m} x}}{n_{m}^{r_{m}}}\right) \mathrm{d} x\right)^{1 / q} .
\end{aligned}
$$

Summing the associated Dirichlet series, having on mind their Laplace integral representations, by means of (9) we get

$$
\sum_{n_{j} \in \mathbb{N}} \frac{e^{-n_{j} x}}{n_{j}^{r_{j}}}=x \int_{1}^{\infty} e^{-x t_{j}}\left(\sum_{n_{j}=1}^{\left[t_{j}\right]} n_{j}^{r_{j}}\right) \mathrm{d} t_{j}=x \int_{1}^{\infty} \int_{0}^{\left[t_{j}\right]} e^{-x t_{j}} \mathfrak{d}_{u_{j}}\left(u_{j}^{-r_{j}}\right) \mathrm{d} t_{j} \mathrm{~d} u_{j} .
$$

Now, obvious transformations lead to the desired inequality. 


\subsection{Two-dimensional Theorem 1}

Taking $m=2$ in Theorem 1 we deduce the following result.

Corollary 6. Suppose $p_{1}^{-1}+p_{2}^{-1}+q^{-1} \geq 1, \mu>0$ and $\mathbf{a}_{i}, i=1,2$ are nonnegative sequences such that

$$
\left(\Phi_{i}^{1 / q}\left(n_{i}\right) a_{n_{i}}\right)_{n_{i}=1}^{\infty} \in \ell_{p_{i}} \quad(i=1,2) .
$$

Then

$$
\sum_{n_{1}, n_{2} \in \mathbb{N}} \frac{a_{n_{1}} a_{n_{2}}}{\left(\lambda_{1}\left(n_{1}\right)+\lambda_{2}\left(n_{2}\right)\right)^{\mu}} \leq \mathrm{C}_{\mu, q}^{\mathbf{\Phi}}(\mathbf{a}, \boldsymbol{\lambda})\left\|\mathbf{a}_{1} \boldsymbol{\Phi}_{1}^{1 / q}\right\|_{p_{1}}\left\|\mathbf{a}_{2} \boldsymbol{\Phi}_{2}^{1 / q}\right\|_{p_{2}} .
$$

Here the constant $\mathbf{C}_{\mu, q}^{\mathbf{\Phi}}(\mathbf{a}, \boldsymbol{\lambda})$ takes the value

$$
\begin{aligned}
\left(\left(\begin{array}{c}
\mu q+1 \\
2
\end{array}\right)\right. & \int_{\lambda_{1}(1)}^{\infty} \int_{\lambda_{2}(1)}^{\infty} \frac{\mathrm{d} t_{1} \mathrm{~d} t_{2}}{\left(t_{1}+t_{2}\right)^{\mu q+2}} \\
& \left.\times \int_{0}^{\left[\lambda_{1}^{-1}\left(t_{1}\right)\right]} \int_{0}^{\left[\lambda_{2}^{-1}\left(t_{2}\right)\right]} \mathfrak{d}_{u_{1}}\left(1 / \Phi_{1}\left(u_{1}\right)\right) \mathfrak{d}_{u_{2}}\left(1 / \Phi_{2}\left(u_{2}\right)\right) \mathrm{d} u_{1} \mathrm{~d} u_{2}\right)^{1 / q} .
\end{aligned}
$$

4.4. $m=2, \Phi_{i}\left(n_{i}\right)=n_{i}^{r_{i}}, \lambda_{i}=\mathcal{I}$

Corollary 7. Suppose $p_{1}^{-1}+p_{2}^{-1}+q^{-1} \geq 1, \mu>0, \Phi_{i}, i=1,2$ positive monotone increasing functions, $\mathbf{a}_{i}, i=1,2$ are nonnegative sequences such that

$$
\left(\Phi_{i}^{1 / q}\left(n_{i}\right) a_{n_{i}}^{r_{i} / q}\right)_{n_{i}=1}^{\infty} \in \ell_{p_{i}} \quad(i=1,2)
$$

and $\lambda_{1}, \lambda_{2}$ are positive monotone increasing functions satisfying (5). Then

$$
\sum_{n_{1}, n_{2}=1}^{\infty} \frac{a_{n_{1}} a_{n_{2}}}{\left(n_{1}+n_{2}\right)^{\mu}} \leq \mathrm{C}_{\mu, q}^{\mathbb{N}^{2}}(\mathbf{a}, \mathcal{I})\left\|\mathbf{a}_{1} \boldsymbol{n}_{1}^{r_{1} / q}\right\|_{p_{1}}\left\|\mathbf{a}_{2} \boldsymbol{n}_{2}^{r_{2} / q}\right\|_{p_{2}},
$$

where the inequality's constant factor $\mathrm{C}_{\mu, q}^{\mathbb{N}^{2}}(\mathbf{a}, \mathcal{I})$ equals

$$
\left(\left(\begin{array}{c}
\mu q+1 \\
2
\end{array}\right) \int_{1}^{\infty} \int_{1}^{\infty} \int_{0}^{\left[t_{1}\right]} \int_{0}^{\left[t_{2}\right]} \frac{\mathfrak{d}_{u_{1}}\left(u_{1}^{-p_{1}}\right) \mathfrak{d}_{u_{2}}\left(u_{2}^{-p_{2}}\right)}{\left(t_{1}+t_{2}\right)^{\mu q+2}} \mathrm{~d} t_{1} \mathrm{~d} t_{2} \mathrm{~d} u_{1} \mathrm{~d} u_{2}\right)^{1 / q} .
$$

\section{References}

[1] J. M. Borwein, Hilbert's inequality and Witten's zeta-function, Amer. Math. Monthly 115 (2008), no. 2, 125-137.

[2] O. Espinosa and V. H. Moll, The evaluation of Tornheim double sums. I, J. Number Theory 116 (2006), no. 1, 200-229.

[3] G. H. Hardy, J. E. Littlewood, and Gy. Pólya, Inequalities, Cambridge University Press, Cambridge, 1934.

[4] J. L. W. V. Jensen, Sur les fonctions convexes et les inégalités entre les valeurs moyennes, Acta Math. 30 (1906), no. 1, 175-193. 
546 BISERKA DRAŠČIĆ BAN, JOSIP PEČARIĆ, IVAN PERIĆ, AND TIBOR POGÁNY

[5] K. Matsumoto, On Mordell-Tornheim and other multiple zeta-functions, Proceedings of the Session in analytic number theory and Diophantine equations (Bonn, January-June 2002.) D.R. Heath-Brown and B. Z. Moroz (eds.), Bonner Mathematische Schriften Nr. 360 (Bonn, 2003), no. 25, 17pp.

[6] D. S. Mitrinović, Analitičke nejednakosti, Građevinska knjiga, Beograd, 1970.

[7] T. K. Pogány, Hilbert's double series theorem extended to the case of non-homogeneous kernels, J. Math. Anal. Appl. 342 (2008), no. 2, 1485-1489.

[8] T. K. Pogány, H. M. Srivastava, and Ž. Tomovski, Some families of Mathieu a-series and alternating Mathieu a-series, Appl. Math. Comput. 173 (2006) 69-108.

[9] M. V. Subbarao and R. Sitaramachandra Rao, On some infinite series of L. J. Mordell and their analogues, Pacific J. Math. 119 (1985), no. 1, 245-255.

[10] L. Tornheim, Harmonic double series, Amer. J. Math. 72 (1950), 303-314.

[11] H. Tsumura, On certain polylogarithmic double series, Arch. Math. (Basel) 88 (2007), no. $1,42-51$.

[12] _ On functional relations between the Mordell-Tornheim double zeta functions and the Riemann zeta function, Math. Proc. Cambridge Philos. Soc. 142 (2007), no. 3, 395-405.

Biserka DRAŠČIĆ BAN

FACUlTy of Maritime Studies

UNIVERSITY OF RIJEKA

51000 Rijeka, Studentska 2, Croatia

E-mail address: bdrascic@pfri.hr

Josip PEČARIĆ

Faculty of Textile Technology

UNIVERSITY OF ZAGREB

10000 Zagreb, Pierottijeva 6, Croatia

E-mail address: pecaric@mahazu.hazu.hr

IVAN PERIĆ

Faculty of Food Technology and Biotechnology

UNIVERSITY OF ZAGREB

10000 Zagreb, Pierottijeva 6, Croatia

E-mail address: iperic@pbf.hr

Tibor Pogány

FaCUlty of Maritime Studies

UNIVERSITY OF RIJEKA

51000 Rijeka, Studentska 2, Croatia

E-mail address: poganj@pfri.hr 